\title{
Nghiên cứu bào chế viên nén Acid Nicotinic giải phóng kéo dài 24 giờ
}

\author{
Phạm Thị Minh Huệ, ${ }^{1, *}$, Nguyễn Văn Bạch ${ }^{2}$, Sonekeo Phommasone ${ }^{3}$ \\ ${ }^{I}$ Truòng Đại học Duoợc Hà Nội, 13-15 Lê Thánh Tông, Hà Nội, Việt Nam \\ ${ }^{2}$ Học Viện Quân Y, 160 Phùng Hung, Hà Đông, Hà Nội, Việt Nam \\ ${ }^{3}$ Cục Quân y Quân đội Nhân dân Lào \\ Nhận ngày 26 tháng 5 năm 2016 \\ Chỉnh sửa ngày 08 tháng 9 năm 2016; Chấp nhận đăng ngày 10 tháng 6 năm 2017
}

\begin{abstract}
Tóm tắt: Niacin (acid nicotinic) là một vitamin rất dễ tan trong nước, được sử dụng để hạ lipid máu. Dang thuốc giải phóng kéo dài chứa niacin được nghiên cứu phát triển nhằm đạt được nồng độ dược chất hằng định trong máu, giảm tác dụng không mong muốn và tăng tuân thủ của người bệnh. Nghiên cứu này nhằm mục tiêu bào chế viên nén niacin giải phóng kéo dài 24 giờ dạng cốt thân nước với tá dược HPMC bằng phương pháp tạo hạt ướt. Kết quả nghiên cứu cho thấy, tốc độ giải phóng dược chất từ viên phụ thuộc vào tỷ lệ $\mathrm{HPMC}$ trong công thức. Bằng phương pháp tối ưu hoá, đã lựa chọn được công thức bào chế viên niacin giải phóng kéo dài 24 giờ đạt độ hoà tan theo USP 37. Quá trình giải phóng dược chất từ viên nghiên cứu theo cơ chế khuếch tán và tuân theo động học 1 .
\end{abstract}

Tù khóa: Niacin, giải phóng kéo dài, cốt thân nước, HPMC.

\section{1. Đặt vấn đề}

Niacin (acid nicotinic) là một dược chất được sử dụng để hạ cholesterol máu và điều trị một số bệnh liên quan đến thiếu niacin. Niacin dễ tan trong nước, hấp thu hoàn toàn qua đường tiêu hoá, thời gian bán thải ngắn (khoảng 45 phút) nên khi dùng ở dạng thuốc quy ước thường phải dùng nhiều lần trong ngày, dễ kích ứng đường tiêu hoá và hiệu quả điều trị không cao [2]. Nghiên cứu bào chế dạng thuốc giải phóng kéo dài (GPKD) chứa acid nicotinic đã thu hút nhiều nhà khoa học trên thế giới $[4,5]$. Tuy nhiên tại Việt Nam, các nghiên cứu bào chế niacin giải phóng kéo dài còn chưa nhiều, dạng viên nén giải phóng kéo dài trên thị trường là thuốc nhập ngoại với giá thành cao. Mục tiêu

\footnotetext{
Tác giả liên hệ. ĐT.: 84-982152969.

Email: phamminhhuehup@gmail.com

https://doi.org/10.25073/2588-1132/vnumps.4059
}

của nghiên cứu là thiết kế công thức bào chế viên nén acid nicotinic 500 mg GPKD 24 giờ dạng cốt thân nước đạt độ hòa tan theo tiêu chuẩn USP 37.

\section{Nguyên liệu và phương pháp nghiên cứu}

Nguyên liệu

Acid nicotinic (Trung quốc, đạt TC(TC) USP), hydroxy propyl methyl cellulose (HPMC) K100M (Đức, đạt tiêu chuẩn TC USP; Avicel PH102, lactose monohydrat, magnesi stearat đạt tiêu chuẩn $\mathrm{BP}$; các dung môi và nguyên liệu khác đạt TC DĐVN IV hoặc tinh khiết phân tích.

\section{Phương pháp nghiên cứu}

Phuơng pháp bố trí thí nghiệm 
Viên nén Acid nicotinic GPKD thiết kế theo hệ cốt thân nước có thành phần cơ bản gồm: acid nicotinic $500 \mathrm{mg}$, HPMC K100M, Avicel PH102, lactose, magnesi stearat.

Bố trí thí nghiệm với các yếu tố cố định: acid nicotinic $500 \mathrm{mg}$, magnesi stearat $16 \mathrm{mg}$, khối lượng viên $800 \mathrm{mg}$. Các yếu tố thay đổi: tỷ lệ HPMC K100M, tỷ lệ Avicel, lực gây vỡ viên (LGVV). Sử dụng phần mềm Modde 8.0 để thiết kế thí nghiệm với các biến độc lập được ghi ở bảng 1 .

Bảng 1. Các biến độc lập và khoảng biến thiên

\begin{tabular}{lccccc}
\hline \multirow{2}{*}{ Các biến độc lập } & Ký & \multicolumn{4}{c}{ Mức biến thiên } \\
\cline { 3 - 6 } & hiệu & $\begin{array}{c}\text { Mức trên } \\
(+1)\end{array}$ & $\begin{array}{c}\text { Mức cơ sở } \\
(0)\end{array}$ & $\begin{array}{c}\text { Mức dưới } \\
(-1)\end{array}$ & $\begin{array}{c}\text { Khoảng } \\
\text { biến thiên }\end{array}$ \\
\hline HPMC K100M (mg) & X1 & 240 & 180 & 120 & 60 \\
LGVV (kP) & X2 & 12 & 10 & 8 & 2 \\
Avicel (mg) & X3 & 40 & 28 & 16 & 12 \\
\hline
\end{tabular}

Các thí nghiệm được bố trí như bảng 2 .

Bảng 2. Các công thức (CT) thực nghiệm

\begin{tabular}{cccccccccc}
\hline CT & $\begin{array}{c}\text { HPMC } \\
\text { K100M } \\
(\mathrm{mg})\end{array}$ & $\begin{array}{c}\text { Avicel } \\
\text { PH102 } \\
(\mathrm{mg})\end{array}$ & $\begin{array}{c}\text { Lacto } \\
\text { se } \\
(\mathrm{mg})\end{array}$ & $\begin{array}{c}\text { Lực gây } \\
\text { vỡ viên } \\
(\mathrm{kP})\end{array}$ & $\mathrm{CT}$ & $\begin{array}{c}\text { HPMC } \\
\text { K100M } \\
(\mathrm{mg})\end{array}$ & $\begin{array}{c}\text { Avicel } \\
\text { PH102 } \\
(\mathrm{mg})\end{array}$ & $\begin{array}{c}\text { Lactose } \\
(\mathrm{mg})\end{array}$ & $\begin{array}{c}\text { Lực gây } \\
\text { vỡ viên } \\
(\mathrm{kP})\end{array}$ \\
\hline N1 & 120 & 16 & 148 & 8 & $\mathrm{~N} 13$ & 160 & 40 & 84 & 10 \\
N2 & 240 & 16 & 28 & 8 & $\mathrm{~N} 14$ & 180 & 28 & 76 & 10 \\
N3 & 240 & 16 & 28 & 8 & $\mathrm{~N} 15$ & 120 & 16 & 148 & 12 \\
N4 & 120 & 40 & 124 & 8 & $\mathrm{~N} 16$ & 240 & 16 & 28 & 12 \\
N5 & 240 & 40 & 4 & 8 & $\mathrm{~N} 17$ & 120 & 40 & 124 & 12 \\
N6 & 240 & 40 & 4 & 8 & $\mathrm{~N} 18$ & 240 & 40 & 4 & 12 \\
N7 & 120 & 32 & 132 & 8 & $\mathrm{~N} 19$ & 240 & 40 & 4 & 12 \\
N8 & 160 & 16 & 108 & 8 & $\mathrm{~N} 20$ & 120 & 32 & 132 & 12 \\
N9 & 180 & 28 & 76 & 8 & $\mathrm{~N} 21$ & 200 & 16 & 68 & 12 \\
N10 & 120 & 16 & 148 & 10 & $\mathrm{~N} 22$ & 180 & 28 & 76 & 12 \\
N11 & 120 & 40 & 124 & 10 & $\mathrm{~N} 23$ & 180 & 28 & 76 & 12 \\
N12 & 240 & 24 & 20 & 10 & $\mathrm{~N} 24$ & 180 & 28 & 76 & 12 \\
\hline
\end{tabular}

Phuơng pháp bào chế viên nén acid nicotinic GPKD

Các bước bào chế như sau: Trộn bột kép dược chất và tá dược, thêm ethanol vừa đủ tạo thành khối ẩm, ủ trong 30-45 phút. Xát hạt qua rây có kích thước $1 \mathrm{~mm}$. Sấy cốm ở nhiệt độ từ $50-60^{\circ} \mathrm{C}$ đến khi còn độ ẩm từ $2-3 \%$. Trộn tá dược trơn và dập viên. Mỗi mẻ dập 100 viên.

Phuoong pháp đánh giá viên

- Xác định độ cứng của viên: Thông qua đo lực gây vỡ viên (LGVV) Được tiến hành trên máy đo lực gây vỡ viên ERWEKA. Tiến hành đo 20 viên, tính giá trị trung bình và độ lệch chuẩn. Yêu cầu sai số $\mathrm{LGVV} \pm 0,05 \mathrm{kP}$ so với bảng bố trí thí nghiệm.

- Định lượng: Bằng phương pháp HPLC (Hệ thống HPLC Agilent Technologies 1200 Series) theo Dược điển Mỹ 37 [7], thay đổi để phù hợp với điều kiện nghiên cứu.

Điều kiện HPLC: Pha tĩnh: Cột sắc ký 4,6 $\mathrm{mm} \times 15 \mathrm{~cm}$ được nhồi pha tĩnh L8 $(5 \mu \mathrm{m})$. Pha động: hỗn hợp dung dịch methanol: nước với

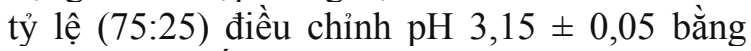
acid acetic, tốc độ dòng: $0,6 \mathrm{ml} /$ phút. Detector UV tại bước sóng $260 \mathrm{~nm}$. Thể tích tiêm là $10 \mu \mathrm{l}$. 
Thử hòa tan: theo chuyên luận "Niacine extended-release tablets" của USP 37 [7]. Thiết bi (Erweka DT-708LH): máy cánh khuấy, tốc độ khuấy: 100 vòng/phút. Môi trường hòa tan: 900 $\mathrm{ml}$ nước cất, nhiệt độ môi trường thử: $37^{\circ} \mathrm{C}$ $\pm 0,5^{\circ} \mathrm{C}$. Các thời điểm lấy mẫu: $1,3,6,9,12$, $15,18,20$ và 24 giờ. Định lượng $\mathrm{DC}$ trong môi trường hoà tan bằng phương pháp HPLC với các điều kiện như ở phần định lượng.

Yêu cầu $\% \mathrm{DC}$ giải phóng theo USP:

\begin{tabular}{lc}
\hline Thời gian (giờ) & \% DC giải phóng \\
\hline 1 & Không lớn hơn 15 \\
3 & $17-32$ \\
6 & $33-48$ \\
9 & $43-63$ \\
12 & $52-77$ \\
20 & Không nhỏ hơn 75 \\
\hline
\end{tabular}

So sánh đồ thị giải phóng duợc chất: Sử dụng chỉ số $\mathrm{f}_{2}$ (similarity factor) thể hiện sự giống nhau giữa hai đồ thị giải phóng $\mathrm{DC}$ được tính theo công thức:

$$
f_{2}=50 . \lg \left\{\left[1+\frac{1}{n} \cdot \sum_{t=1}^{n}\left(R_{t}-T_{t}\right)^{2}\right]^{-0,5} .100\right\}
$$

Trong đó:

n: Số điểm lấy mẫu.

$\mathrm{R}_{\mathrm{t}}$ : $\%$ dược chất hoà tan tại thời điểm $\mathrm{t}$ của mẫu đối chiếu.

Bảng 3. Tỷ lệ (\%) Acid nicotinic giải phóng theo thời gian từ viên nén Acid nicotinic $\operatorname{GPKD}\left(\mathrm{n}=6, \overline{\mathrm{X}}_{ \pm} \mathrm{SD}\right)$
$\mathrm{T}_{\mathrm{t}}$ : \% dược chất hoà tan tại thời điểm $\mathrm{t}$ của mẫu nghiên cứu.

Theo quy định của $F D A$ và EMEA $f_{2} \in[50$; 100] thì 2 đồ thị coi là giống nhau [6].

Đánh giá mô hình động học giải phóng dược chất thông qua một số mô hình như bậc không, bậc 1 (mô hình Wagner), Weibull, Higuchi, Hixson-Crowell, Korsmeyer-Peppas... Trong đó, mô hình động học phù hợp nhất với sự giải phóng dược chất được đánh giá qua tiêu chuẩn $\mathrm{AIC}$ và $\mathrm{R}_{\mathrm{hc}}{ }_{\mathrm{hc}}$ [3] (được tính toán bằng phần mềm MathCAD 15.0).

- Tối ưu hóa công thức: nhờ sự trợ giúp phần mềm INForm 3.1.

\section{Kết quả nghiên cứu}

\subsection{Bào chế và đánh giá các mẫu viên}

Bào chế viên theo công thức ở bảng 2 . Mỗi công thức bào chế 100 viên.

- Hàm lượng dược chất trong các mẫu viên định lượng được từ 95,63 $\pm 1,34 \%$ đến 97,93 $\pm 0,73$ $\%(n=3)$, đạt yêu cầu về giới hạn hàm lượng theo DĐVN IV (90 \% - 110\%).

- Thử hoà tan các mẫu viên, kết quả được trình bày ở bảng 3 .

\begin{tabular}{|c|c|c|c|c|c|c|c|c|c|}
\hline \multirow[b]{2}{*}{ CT } & \multicolumn{9}{|c|}{ Tỷ lệ (\%) DC giải phóng theo thời gian } \\
\hline & 1 giờ & 3 giờ & 6 giờ & 9 giờ & 12 giờ & 15giờ & 18giờ & 20giờ & 24 giờ \\
\hline \multirow{2}{*}{ N1 } & 14,11 & 28,02 & 45,92 & 60,38 & 74,18 & 83,14 & 85,40 & 90,47 & 94,31 \\
\hline & $\pm 1,03$ & $\pm 1,21$ & $\pm 2,20$ & $\pm 1,13$ & $\pm 2,17$ & $\pm 2,08$ & $\pm 1,89$ & $\pm 1,24$ & $\pm 4,13$ \\
\hline \multirow[b]{2}{*}{ N2 } & 10,24 & 22,70 & 37,56 & 52,29 & 64,39 & 66,33 & 77,38 & 82,62 & 87,16 \\
\hline & $\pm 1,09$ & $\pm 1,23$ & $\pm 2,38$ & $\pm 1,36$ & $\pm 2,38$ & $\pm 1,24$ & $\pm 2,51$ & $\pm 2,26$ & $\pm 3,37$ \\
\hline \multirow{2}{*}{ N3 } & 10,31 & 23,02 & 38,86 & 52,45 & 62,32 & 64,51 & 76,37 & 80,51 & 85,52 \\
\hline & $\pm 2,11$ & $\pm 1,28$ & $\pm 1,44$ & $\pm 1,42$ & $\pm 1,24$ & $\pm 1,20$ & $\pm 1,36$ & $\pm 3,47$ & $\pm 2,21$ \\
\hline \multirow{2}{*}{ N4 } & 13,46 & 30,69 & 48,04 & 66,19 & 78,29 & 79,37 & 92,39 & 93,20 & 95,10 \\
\hline & $\pm 2,16$ & $\pm 2,48$ & $\pm 1,57$ & $\pm 1,48$ & $\pm 1,39$ & $\pm 1,32$ & $\pm 1,71$ & $\pm 3,22$ & $\pm 2,08$ \\
\hline \multirow{2}{*}{ N5 } & 9,73 & 22,03 & 35,52 & 50,90 & 62,18 & 62,82 & 75,18 & 75,82 & 87,01 \\
\hline & $\pm 1,09$ & $\pm 1,24$ & $\pm 1,34$ & $\pm 1,28$ & $\pm 1,21$ & $\pm 1,32$ & $\pm 1,63$ & $\pm 3,36$ & $\pm 2,27$ \\
\hline \multirow[b]{2}{*}{ N6 } & 9,71 & 22,15 & 35,36 & 54,13 & 63,65 & 65,00 & 78,81 & 82,12 & 86,27 \\
\hline & $\pm 3,18$ & $\pm 1,32$ & $\pm 1,31$ & $\pm 1,43$ & $\pm 1,26$ & $\pm 1,14$ & $\pm 1,21$ & $\pm 2,11$ & $\pm 3,37$ \\
\hline \multirow{2}{*}{ N7 } & 10,30 & 27,48 & 44,64 & 59,67 & 79,12 & 81,22 & 95,38 & 95,79 & 97,05 \\
\hline & $\pm 2,05$ & $\pm 2,38$ & $\pm 1,42$ & $\pm 1,46$ & $\pm 1,08$ & $\pm 2,21$ & $\pm 1,55$ & $\pm 2,26$ & $\pm 1,19$ \\
\hline
\end{tabular}




\begin{tabular}{|c|c|c|c|c|c|c|c|c|c|}
\hline \multirow{2}{*}{ N8 } & 11,73 & 26,76 & 42,75 & 61,84 & 72,03 & 72,90 & 86,56 & 89,38 & 93,87 \\
\hline & $\pm 1,12$ & $\pm 2,34$ & $\pm 2,42$ & $\pm 2,58$ & $\pm 1,31$ & $\pm 1,23$ & $\pm 1,39$ & $\pm 2,14$ & $\pm 3,41$ \\
\hline \multirow{2}{*}{ N9 } & 10,41 & 24,37 & 41,68 & 54,42 & 67,13 & 75,49 & 80,37 & 86,14 & 92,17 \\
\hline & $\pm 2,06$ & $\pm 1,12$ & $\pm 1,16$ & $\pm 2,65$ & $\pm 1,31$ & $\pm 1,31$ & $\pm 1,52$ & $\pm 2,54$ & $\pm 2,35$ \\
\hline \multirow{2}{*}{ N10 } & 11,91 & 27,98 & 45,72 & 60,70 & 76,54 & 84,86 & 85,95 & 92,17 & 94,93 \\
\hline & $\pm 1,06$ & $\pm 1,12$ & $\pm 1,24$ & $\pm 1,41$ & $\pm 1,20$ & $\pm 1,45$ & $\pm 1,41$ & $\pm 3,36$ & $\pm 2,51$ \\
\hline \multirow{2}{*}{ N11 } & 10,40 & 23,83 & 39,32 & 53,23 & 68,34 & 79,06 & 84,64 & 93,84 & 97,24 \\
\hline & $\pm 3,03$ & $\pm 3,11$ & $\pm 2,26$ & $\pm 1,12$ & $\pm 1,28$ & $\pm 1,29$ & $\pm 2,27$ & $\pm 3,18$ & $\pm 2,26$ \\
\hline \multirow{2}{*}{ N12 } & 9,45 & 22,45 & 35,41 & 47,78 & 59,26 & 67,32 & 71,84 & 80,12 & 86,28 \\
\hline & $\pm 2,02$ & $\pm 1,51$ & $\pm 1,18$ & $\pm 1,08$ & $\pm 1,25$ & $\pm 1,40$ & $\pm 2,27$ & $\pm 3,14$ & $\pm 2,27$ \\
\hline \multirow{2}{*}{ N13 } & 11,33 & 25,70 & 41,29 & 54,13 & 68,81 & 77,13 & 78,09 & 88,16 & 93,53 \\
\hline & $\pm 1,06$ & $\pm 1,23$ & $\pm 1,31$ & $\pm 2,06$ & $\pm 2,30$ & $\pm 2,47$ & $\pm 2,15$ & $\pm 2,31$ & $\pm 1,31$ \\
\hline \multirow{2}{*}{ N14 } & 10,97 & 24,88 & 37,55 & 52,34 & 68,70 & 77,72 & 80,31 & 89,47 & 94,84 \\
\hline & $\pm 2,04$ & $\pm 1,12$ & $\pm 2,30$ & $\pm 2,33$ & $\pm 2,32$ & $\pm 1,15$ & $\pm 1,20$ & $\pm 1,29$ & $\pm 2,43$ \\
\hline \multirow{2}{*}{ N15 } & 11,08 & 20,98 & 34,67 & 46,67 & 59,93 & 68,93 & 74,43 & 81,36 & 91,72 \\
\hline & $\pm 2,03$ & $\pm 2,16$ & $\pm 1,18$ & $\pm 1,31$ & $\pm 1,39$ & $\pm 1,29$ & $\pm 2,16$ & $\pm 1,34$ & $\pm 1,30$ \\
\hline \multirow{2}{*}{ N16 } & 8,26 & 16,52 & 27,76 & 36,51 & 45,72 & 52,61 & 57,84 & 64,26 & 73,12 \\
\hline & $\pm 2,03$ & $\pm 2,08$ & $\pm 2,12$ & $\pm 3,20$ & $\pm 1,28$ & $\pm 1,27$ & $\pm 2,16$ & $\pm 1,25$ & $\pm 1,08$ \\
\hline \multirow{2}{*}{ N17 } & 11,24 & 23,38 & 39,08 & 50,94 & 64,06 & 75,99 & 80,94 & 89,85 & 93,69 \\
\hline & $\pm 2,02$ & $\pm 1,15$ & $\pm 2,20$ & $\pm 1,27$ & $\pm 1,21$ & $\pm 2,27$ & $\pm 1,25$ & $\pm 1,18$ & $\pm 2,08$ \\
\hline \multirow{2}{*}{ N18 } & 7,55 & 16,12 & 23,85 & 32,87 & 37,38 & 54,89 & 64,82 & 67,60 & 74,35 \\
\hline & $\pm 1,03$ & $\pm 2,05$ & $\pm 2,03$ & $\pm 1,11$ & $\pm 1,17$ & $\pm 1,22$ & $\pm 1,20$ & $\pm 2,17$ & $\pm 3,18$ \\
\hline \multirow{2}{*}{ N19 } & 7,75 & 15,06 & 23,98 & 33,53 & 36,86 & 53,53 & 73,05 & 81,77 & 91,68 \\
\hline & $\pm 3,05$ & $\pm 2,04$ & $\pm 1,09$ & $\pm 1,19$ & $\pm 1,43$ & $\pm 1,30$ & $\pm 1,22$ & $\pm 2,46$ & $\pm 3,18$ \\
\hline \multirow{2}{*}{ N20 } & 12,13 & 26,89 & 42,22 & 62,64 & 68,37 & 79,17 & 86,12 & 95,50 & 102,12 \\
\hline & $\pm 3,07$ & $\pm 1,13$ & $\pm 3,25$ & $\pm 1,36$ & $\pm 1,22$ & $\pm 1,11$ & $\pm 1,17$ & $\pm 2,20$ & $\pm 3,62$ \\
\hline \multirow{2}{*}{ N21 } & 12,56 & 25,74 & 43,00 & 54,49 & 67,70 & 79,04 & 80,87 & 89,08 & 90,30 \\
\hline & $\pm 2,03$ & $\pm 1,07$ & $\pm 3,10$ & $\pm 2,17$ & $\pm 1,46$ & $\pm 1,26$ & $\pm 2,20$ & $\pm 2,12$ & $\pm 2,05$ \\
\hline \multirow{2}{*}{ N22 } & 9,84 & 19,76 & 32,87 & 43,94 & 54,99 & 66,02 & 70,99 & 79,76 & 89,60 \\
\hline & $\pm 1,03$ & $\pm 2,06$ & $\pm 2,08$ & $\pm 2,18$ & $\pm 1,22$ & $\pm 1,16$ & $\pm 1,08$ & $\pm 1,08$ & $\pm 2,11$ \\
\hline \multirow{2}{*}{ N23 } & 9,16 & 19,10 & 32,10 & 41,61 & 53,01 & 62,18 & 67,49 & 75,64 & 86,42 \\
\hline & $\pm 3,02$ & \pm 3.09 & $\pm 2,05$ & $\pm 1,12$ & $\pm 1,05$ & $\pm 2,14$ & $\pm 2,17$ & $\pm 3,29$ & $\pm 2,18$ \\
\hline \multirow{2}{*}{ N24 } & 9,62 & 18,94 & 31,73 & 41,53 & 52,83 & 62,40 & 66,75 & 75,32 & 84,66 \\
\hline & $\pm 2,02$ & $\pm 2,08$ & $\pm 2,03$ & $\pm 1,06$ & $\pm 2,13$ & $\pm 2,08$ & $\pm 2,18$ & $\pm 3,43$ & $\pm 3,08$ \\
\hline
\end{tabular}

Nhận xét: tất cả 24 công thức thực nghiệm đều có khả năng kéo dài quá trình giải phóng acid nicotinic. Tuy nhiên, tốc độ giải phóng dược chất từ các công thức là khác nhau do hàm lượng các loại tá dược và độ cứng viên là khác nhau. Đánh giá ảnh hưởng của biến độc lập tới tốc độ giải phóng dược chất (biến phụ thuộc) dựa vào phần mềm INForm 3.1 với yêu cầu đặt ra là hệ số tương quan $\mathrm{R}^{2}$ luyện và $\mathrm{R}^{2}$ thử từ 80 - 100 (số lớp ẩn 1; số nút trong lớp ẩn 2; thuật toán lan truyền ngược RPROP). Kết quả luyện của các công thức thể hiện ở bảng sau:

Bảng 4 . Kết quả $\mathrm{R}^{2}$ luyện và $\mathrm{R}^{2}$ thử của công thức

\begin{tabular}{cccccccccc}
\hline & $\mathrm{Y} 1$ & $\mathrm{Y} 3$ & $\mathrm{Y} 6$ & $\mathrm{Y} 9$ & $\mathrm{Y} 12$ & $\mathrm{Y} 15$ & $\mathrm{Y} 18$ & $\mathrm{Y} 20$ & $\mathrm{Y} 24$ \\
\hline $\mathrm{R}^{2}$ luyện & 79,46 & 86,80 & 90,62 & 89,35 & 94,67 & 91,41 & 90,52 & 81,82 & 75,44 \\
$\mathrm{R}^{2}$ thử & 100 & 100 & 100 & 100 & 100 & 100 & 100 & 100 & 100 \\
\hline
\end{tabular}


4.2. Đánh giá ảnh hưởng của các biến độc lập đến biến phu thuộc

- HPMC K100M: Hàm lượng HPMC $\mathrm{K} 100 \mathrm{M}$ càng tăng thì tỷ lệ giải phóng $\mathrm{DC}$ càng giảm, vì HPMC là polyme thân nước có độ nhớt cao $(100000 \mathrm{cP})$, khi tỷ lệ cao trong viên, khả năng hút nước tạo lớp gel dày đặc sẽ làm chậm sự giải phóng $\mathrm{DC}$ ra môi trường hòa tan. Kết quả được biểu diễn ở hình 1 và 2 .

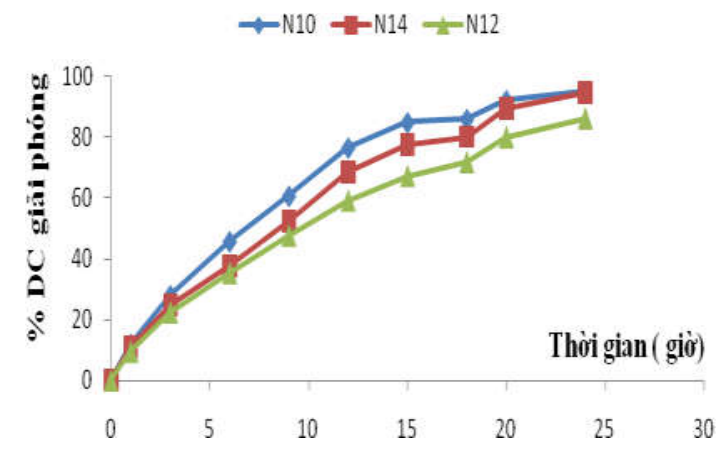

Hình 1. Đồ thị biểu diễn tỷ lệ (\%) Acid nicotinic giải phóng theo thời gian từ các viên bào chế theo $\mathrm{CT}$ $\mathrm{N} 10, \mathrm{~N} 12$ và N14.

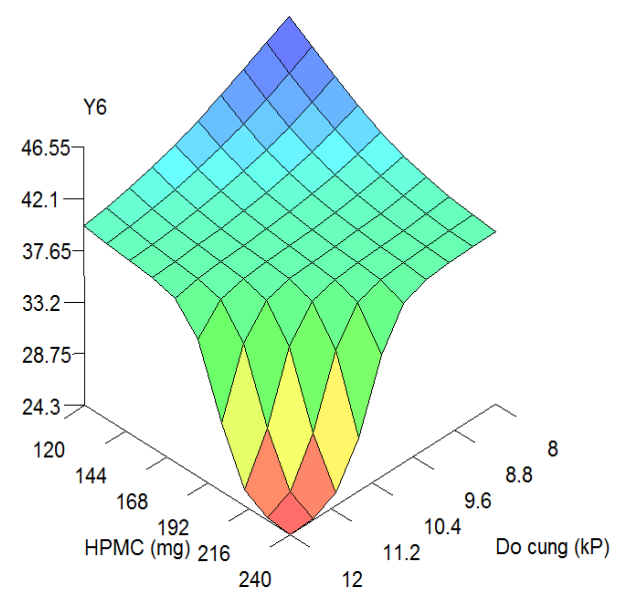

Hình 2. Mặt đáp Y6 theo HPMC K100M và độ cứng viên (Avicel tại tâm $=28 \mathrm{mg}$ ).

Khi tăng đồng thời khối lượng của HPMC $\mathrm{K} 100 \mathrm{M}$ từ $120 \mathrm{mg}$ đến $240 \mathrm{mg}$ và độ cứng từ 8 đến $12 \mathrm{kP}$ thì tỷ lệ $(\%) \mathrm{DC}$ giải phóng giảm (hình 2). Khi tăng đồng thời khối lượng của Avicel từ $16 \mathrm{mg}$ đến $40 \mathrm{mg}$ và độ cứng từ 8 đến
$12 \mathrm{kP}$ thì tỷ lệ (\%) DC giải phóng giảm xuống (hình 3).

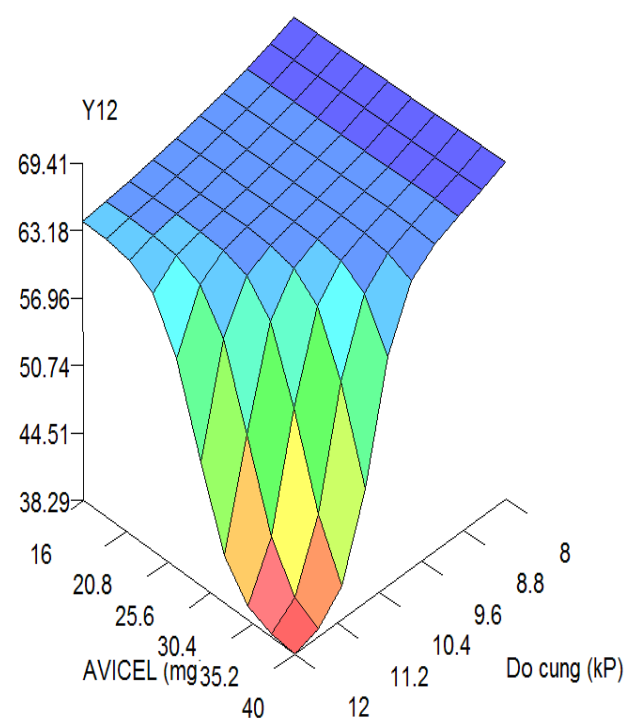

Hình 3. Mặt đáp Y6 theo Avicel và độ cứng viên (HPMC tại tâm $180 \mathrm{mg}$ ).

Avicel là tá dược độn, có khả năng làm viên chắc, mặc dù có khả năng trương nở nhưng do HPMC có độ nhớt rất cao nên tác động của tá dược này đển giải phóng DC không rõ rệt. Trong các công thức nghiên cứu, lactose làm tá dược độn để vừa đủ khối lượng viên, hầu như không ảnh hưởng đến giải phóng dược chất. Đối với viên nén, lực nén ảnh hưởng đến hệ thống vi mao quản do đó có thể ảnh hưởng tới quá trình hút nước và rã. Tuy nhiên đối với hệ cốt thân nước sử dụng polyme có độ nhớt cao, quá trình hút nước chủ yếu phụ thuộc vào tốc độ trương nở của tá dược và do hàng rào gel dày đặc, quá trình giải phóng dược chất xảy ra theo cơ chế khuếch tán.

\subsection{Tối ưu hóa công thức bào chế:}

Từ các số liệu thực nghiệm, công thức tối ưu tìm được các giá trị X1 = $180 \mathrm{mg} ; \mathrm{X} 2=10$ $\mathrm{kP} ; \mathrm{X} 3=24 \mathrm{mg}$

Tiến hành bào chế theo công thức tối ưu và đánh giá độ hòa tan, so sánh với số liệu dự đoán. Kết quả thể hiện ở bảng 5 . 
Bảng 5. Tỷ lệ (\%) Acid nicotinic giải phóng theo thời gian từ viên nén acid nicotinic GPKD (n=12, $\overline{\mathrm{X}} \pm \mathrm{SD})$

\begin{tabular}{|c|c|c|c|c|c|c|c|c|c|}
\hline \multirow{2}{*}{ Mẫu } & \multicolumn{9}{|c|}{ Thời gian (giờ) } \\
\hline & 1 & 3 & 6 & 9 & 12 & 15 & 18 & 20 & 24 \\
\hline CT tối ưu & $12,73 \pm 2,02$ & $25,75 \pm 1,09$ & $42,53 \pm 2,06$ & $54,64 \pm 3,04$ & $68,75 \pm 1,79$ & $78,32 \pm 1,63$ & $84,86 \pm 2,62$ & $88,20 \pm 1,59$ & $94,89 \pm 2,54$ \\
\hline $\begin{array}{l}\text { Dự đoán } \\
\text { của } \\
\text { INform }\end{array}$ & 12,09 & 29,02 & 39,40 & 51,72 & 65,71 & 78,90 & 86,77 & 92,52 & 96,07 \\
\hline USP 37 & $<15$ & $17-32$ & $33-48$ & $43-63$ & $52-77$ & & & $>75$ & \\
\hline
\end{tabular}

Nhận xét: Tỷ lệ phần trăm giải phóng dược chất tại các thời điểm thỏa mãn yêu cầu của bài toán tối ưu và yêu cầu của USP 37 . Chỉ số $\mathrm{f}_{2}$ so sánh đồ thị hoà tan của viên nén acid nicotinic GPKD bào chế theo công thức tối ưu và dự đoán là 76,26 (nằm trong khoảng 50-100).

Đánh giá động học giải phóng của viên acid nicotinic GPKD bào chế theo công thức tối ưu, so sánh với các mô hình khác được trình bày ở bảng 6 và hình 4 .
Mô hình bậc 1 (Wagner) có giá trị AIC là nhỏ nhất và $\mathrm{R}_{\mathrm{hc}}^{2}$ là lớn nhất $(\mathrm{AIC}=43,696$ và $\left.\mathrm{R}_{\mathrm{hc}}^{2}=0,996\right)$, phù hợp hơn cả để giải thích cơ chế giải phóng $\mathrm{DC}$ từ viên. Đây là mô hình đặc trưng cho DC dễ tan trong nước bào chế với cốt thân nước có quá trình giải phóng DC theo cơ chế khếch tán qua lớp gel trương nở của tá dược HPMC (Hình 4).

Bảng 6. Giá trị $\mathrm{AIC}$ và $\mathrm{R}_{\mathrm{hc}}^{2}$ theo từng mô hình động học của công thức tối ưu

\begin{tabular}{ccccccccc}
\hline & Bậc 0 & Wagner & Weibull & Higuchi & Hixson crowell & Korsmeyer peppas & Hopfenberg & Quadratic \\
\hline AIC & 79,28 & 43,69 & 45,695 & 60,048 & 52,463 & 58,774 & 45,695 & 54,608 \\
$\mathrm{R}^{2} \mathrm{hc}$ & 0,917 & 0,996 & 0,996 & 0,984 & 0,992 & 0,987 & 0,996 & 0,991 \\
\hline
\end{tabular}

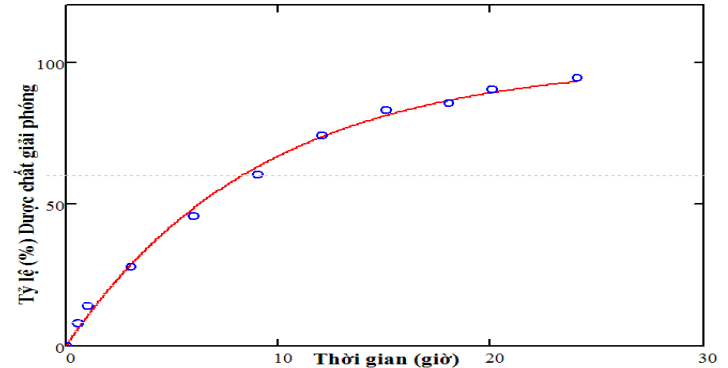

Hình 4. Đồ thị biểu diễn tỷ lệ (\%) dược chất giải phóng từ công thức tối ưu theo mô hình Wagner (bậc 1) [6].

\subsection{Bàn luận}

Dạng cốt thân nước là một trong các hệ kiểm soát giải phóng dược chất kéo dài được quan tâm nghiên cứu nhiều trong những năm gần đây [5]. Cốt thân nước sử dụng tá dược HPMC có nhiều ưu điểm như dễ bào chế viên nén do chịu nén và trơn chảy tốt; có độ nhớt xác định; tương hợp tốt với nhiều dược chất và tá dược khác. Việc sử dụng HPMC K100M cho viên nén acid nicotinic giải phóng kéo dài 24 giờ là phù hợp do niacin là dược chất dễ tan trong nước, hàm lượng cao, thời gian giải phóng $\mathrm{DC}$ cần kéo dài [5]. Phương pháp bố trí thí nghiệm và tối ưu hoá công thức sử dụng trong nghiên cứu cho phép thống kê các xu hướng ảnh hưởng, tiết kiệm số lượng thực nghiệm và dự đoán được công thức tối ưu sát với thực nghiệm. Mô hình giải phóng dược chất từ viên tuân theo mô hình động học bậc 1 , nằm trong giới hạn độ hoà tan theo USP, phù hợp với cơ chế khếch tán dược chất xảy ra đồng thời với quá trình hút nước trương nở gel HPMC. Kết quả phân tích động học giải phóng dược chất còn giúp dự đoán độ hoà tan liên quan tới lựa chọn tá dược phù hợp với độ tan của mỗi dược chất, nhằm ứng dụng cho các nghiên cứu sâu hơn về mối tương quan SKD in vitro- in vivo sau này. 


\section{Kết luận}

Đã xây dựng được công thức viên nén acid nicotinic $500 \mathrm{mg}$ GPKD 24 giờ bào chế bằng phương pháp tạo hạt ướt ở qui mô phòng thí nghiệm. Viên bào chế với công thức lựa chọn có độ hòa tan đạt tiêu chuẩn USP37 và giống với dự đoán từ mô hình. Quá trình giải phóng $\mathrm{DC}$ từ viên được kiểm soát bởi polyme thân nước có độ nhớt cao theo cơ chế khếch tán $\mathrm{DC}$ và tuân theo động học bậc 1 . Với công thức lựa chọn có thể áp dụng để nâng cấp qui trình bào chế ở qui mô lớn hơn nhằm áp dụng vào thực tiễn.

\section{Tài liệu tham khảo}

[1] Bộ Y tế, Dược điển Việt Nam IV, NXB Y học, 2009.

[2] Harol E. Bays, Carlos A. Dujovne, (2003), "Comparison of one - daily, Niacin extendedrelease/ Lovastatin with Standard doses of
Atorvastatin and Simvastatin (The advicor Versus othe cholesterol-Modulating Agents Trial Evaluation)", Am J Cardiol., 91, pp. 667-672.

[3] Juergen Siepmann, F. Siepmann (2008), "Mathematical modeling of drug delivery", International Journal of Pharmaceutics, 364, pp. 328-343.

[4] Patel D.N., Ghelani T.K. (2011), "Formulation and evaluation of once daily niacin extended release matrix tablets", Pharma Science Monitor, 2(4), pp.145-153.

[5] Rezowanur Rahman, Sheikh Tasnim Jahan (2010), "Preparation and evaluation of mucoadhesive hydrophilic hydroxy propyl methyl cellulose based extended release matrix tablets of Niacin (Nicotinic acid)", American Journal of Scientific and Industrial Research, pp. 558-564.

[6] Paulo Costa, José Manuel Sousa Lobo (2001), "Modeling and comparison of dissolution profiles", European Journal of Pharmaceutical Sciences, 13, pp. 123-133.

[7] USP 37, pp. 3980-3981.

\title{
Preparation of 24 - hrs Sustained Release Tablets of Nicotinic Acid
}

\author{
Pham Thi Minh Hue ${ }^{1}$, Nguyen Van Bach ${ }^{2}$, Sonekeo Phommasone ${ }^{3}$ \\ ${ }^{1}$ Hanoi University of Pharmacy, 13-15 Le Thanh Tong, Hanoi, Vietnam \\ ${ }_{2}^{2}$ Military Medical University, No. 160, Phung Hung Street, Ha Dong District, Hanoi, Vietnam \\ ${ }^{3}$ Lao People's Army Medical Department
}

\begin{abstract}
Niacin (nicotinic acid) is a highly water-soluble vitamin, which has been used as a lipidlowering agent. Sustained release (SR) niacin tablet was developed in oder to maintain a constant drug concentration for a specific period of time with minimum side effects. In this study, the once daily extended release matrix tablets of niacin using a hydrophilic polymer of HPMC K100M as matrix excipient were prepared by wet granulation method. The results of the dissolution study indicated that the release of the drug was found to be dependent on the proportions of HPMC used in the tablets. The dissolution profile of the optimal formula was similar to that of the predict and attained the requirements of USP 37. The dug incorporated in the matrix released by diffusion and fitted the first order kinetic.
\end{abstract}

Keyword: Niacin, sustained release, hydrophilic matrix, HPMC. 